\title{
Ubiquitous Statistical Acceleration in the Solar Wind
}

\author{
L. A. Fisk, G. Gloeckler, T. H. Zurbuchen, and N. A. Schwadron \\ Department of Atmospheric, Oceanic, and Space Science \\ University of Michigan, Ann Arbor, MI 48109
}

\begin{abstract}
One of the more interesting observations by ACE is the ubiquitous presence of higher energy tails on the distribution functions of solar wind and pickup ions. The tails occur continuously in the slow solar wind, but less so in fast wind. Their presence is not correlated with the passage of shock waves. It is pointed out that statistical acceleration by transit-time damping of propagating magnitude fluctuations in the magnetic field of the solar wind is a likely mechanism to yield the observed tails.
\end{abstract}

\section{INTRODUCTION}

In companion papers in this Proceedings, Gloeckler et al. (1) and Zurbuchen et al. (2) provide stunning new observations of the ubiquitous presence of pronounced tails on the distributions of ions in the solar wind, both of solar wind origin and pickup ions. Shown in Figure 1 is a summary of these observations, for hydrogen and singly and doubly charged helium, as seen by the SWICS instrument on ACE. Similar distribution functions are observed from Ulysses/SWICS during its passage through the solar equatorial plane. There are clear tails on all three distributions, extending from essentially solar wind thermal energies, up to the limits of these instruments; in the case of hydrogen up to ten times the solar wind flow speed. From ACE at $1 \mathrm{AU}$, the hydrogen tail is due to accelerated solar wind protons. From Ulysses at 4-5 $\mathrm{AU}$, interstellar pickup hydrogen is present and accelerated. Singly charged helium is an interstellar pickup ion, and is accelerated at the locations of both $\mathrm{ACE}$ and Ulysses. The acceleration of the pickup ions indicates that the tails on the distribution functions are created in the solar wind, and are not the remnant of some coronal process.

The most significant point concerning these tails is that in the slow solar wind, near the solar equatorial plane, the tails on the thermal distributions of solar wind and pickup ions are always present. They do not appear to occur as commonly at the higher latitudes seen by Ulysses (3). However, they are ubiquitous in the slow solar wind. Most important for deciding on an explanation for their origin, they are unrelated to the presence or passage of any shock wave. These tails are continuous from thermal speeds of order the solar wind flow speed, up to ten times that value. At the lower speeds, the particles cannot propagate far in the solar wind, but rather they are transported simply by convection with the solar wind. Such particles do not arrive at earth from some distant shock such as the standing shocks that bound Co-rotating Interaction Regions (CIRs) since they lack the upstream speed to do so. We are forced to conclude, therefore, that these particles are not generated by shocks.

The only alternative acceleration mechanism is statistical acceleration, in which the particles interact with random electric fields present in the solar wind. We will conclude in this paper that only transit time damping of propagating magnitude fluctuations in the solar wind, as proposed by Fisk (4), is likely to cause the observed acceleration. There are continuous sources of magnitude fluctuations in the solar wind, some of which are described, and the transit time damping mechanism is quite efficient.

With regard to higher-energy particles, in the $\mathrm{MeV}$ range, shock acceleration is undoubtedly the preferred mechanism. There is a well known correlation of accelerated particles with shocks in CIRs (5). Although the particles observed in the tails of the thermal distributions do not originate in shocks, they could serve as the particles that are injected into a shock acceleration mechanism. Indeed, the particles 
well up on the tails, with higher speeds, should be able to be readily injected into a standard diffusive shock acceleration mechanism.

We begin by reviewing the general properties of statistical acceleration, and the requirements for the efficiency of the acceleration that is needed to produce the observed tails. We then consider the statistical acceleration that accompanies pitch-angle scattering by Alfven waves, and from transit-time damping of magnetosonic waves, and conclude only the latter is viable. Finally, we discuss possible sources of magnetosonic waves in the solar wind.

\section{GENERAL PROPERTIES OF STATISTICAL ACCELERATION}

Statistical acceleration can always be expressed as a diffusion in momentum space, by, e.g., the following equation:

$$
\begin{aligned}
\mathbf{V} \cdot \nabla f= & \nabla \cdot \kappa \cdot \nabla f+\frac{1}{p^{2}} \frac{\partial}{\partial p}\left(p^{2} D_{p p} \frac{\partial f}{\partial p}\right) \\
& +\frac{p}{3} \nabla \cdot \mathbf{V} \frac{\partial f}{\partial p}
\end{aligned}
$$

The equation is written as a steady state. The term on the left side describes convection with the solar wind velocity, V. The first term on the right describes spatial diffusion; the second term on the right is diffusion in momentum, or the statistical acceleration; and finally the third term on the right is due to adiabatic deceleration in the solar wind. The spatial diffusion term needs to be treated with some caution in the case of the low velocity particles shown in Figure 1. Spatial diffusion is usually derived in the limit that the particle speed is large compared to the plasma flow speed. The statistical acceleration term is probably reasonably accurate so long as the particle speed is larger than the characteristic speeds of the waves or turbulence with which the particles interact.

Statistical acceleration is governed by a diffusion coefficient in momentum space $D_{p p}$. The statistical acceleration must be expressed only in terms of derivatives of the distribution function in momentum, because the Vlasov or Liouville's equation that was the origin of equation (1) contained only derivatives of the distribution with momentum.

Statistical acceleration can also be described as a diffusion in energy space, with diffusion coefficient, $D_{T T}$. The two diffusion coefficients are of course related to each other, as $D_{T T}=v^{2} D_{p p}$. In the case of diffusion in energy, there is also a mean change in energy. There are no negative energies, and so as particles diffuse in momentum, the mean energy increases at the rate $D_{T T} / 2 T$. When evaluating a statistical acceleration mechanism any of these various forms can be used since they are all interrelated.

To create the tails shown in Figure 1, the diffusion in momentum or energy must have a characteristic time of order the solar wind convection time, or equivalently,

$$
\frac{T^{2}}{D_{T T}} \approx \frac{r}{V} \approx 3.75 \times 10^{5} \mathrm{sec}
$$

As can be seen in equation (1), the time change in the distribution function due to statistical acceleration will then be comparable to the time during which particles are convected through the heliosphere, and a significant tail can be produced at earth.

\section{STATISTICAL ACCELERATION BY PITCH ANGLE SCATTERING}

One possibility for a statistical acceleration mechanism is pitch-angle scattering from moving magnetic irregularities, as was discussed by, e.g. Jokipii (6) and Wibberentz and Beuermann (7). The concept here is quite simple. Particles are scattered in

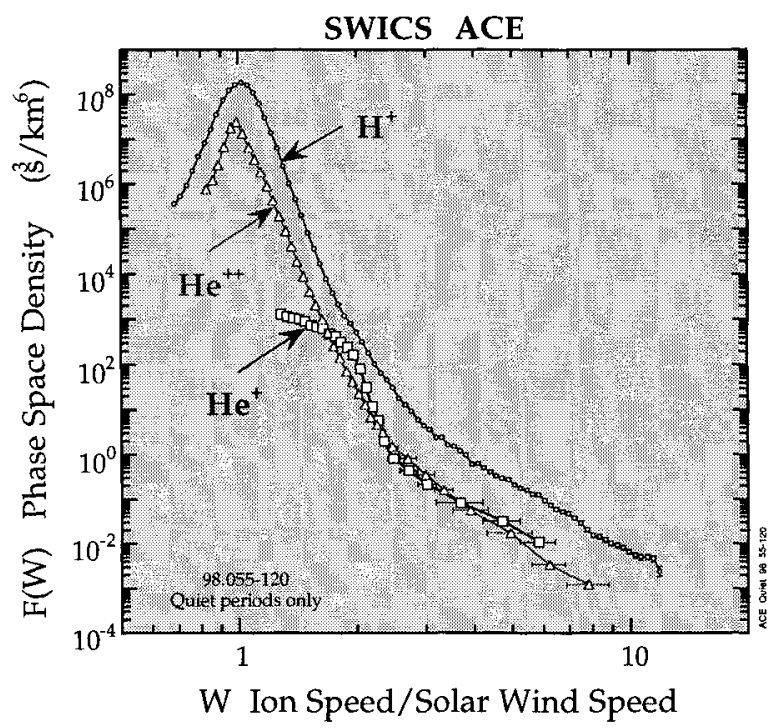

Figure 1. The distribution functions of $\mathrm{H}^{+}, \mathrm{He}^{+}$, and $\mathrm{He}^{++}$, as seen by the SWICS instrument on ACE (1). 
pitch angle presumably from Alfven waves, which move randomly. Like tennis balls bouncing off a moving wall, the reversal of the particle's velocity in the frame moving with the wave causes an energy change in the solar wind frame. As discussed by Jokipii (6) and by Fisk (4), the relevant diffusion coefficient in energy space is

$$
D_{T T}=V_{\text {Alfuen }}^{2} T^{2} / \kappa_{\text {parallel }}
$$

Here $V_{\text {Alfven }}$ is the Alfven speed and $\kappa_{\text {paralle }}=v \lambda / 3$ is the diffusion coefficient for pitch-angle scattering parallel to the mean magnetic field, with $\lambda$ the mean free path.

The difficulty with this mechanism is the mean free path required for this diffusion coefficient to meet our criteria of being important on the time scale for convection. The value for the mean free path required to satisfy equation (2) is

$$
\lambda=\frac{3 V_{\text {Alfven }}^{2} r}{v V}<5 \times 10^{-2} \mathrm{AU}
$$

Recall that pick up ions in particular are known to have mean free paths of order $1 \mathrm{AU}(8)$, i.e., very little pitch angle scattering. Statistical acceleration by pitch angle scattering is not a viable acceleration mechanism in this instance.

\section{STATISTICAL ACCELERATION BY TRANSIT-TIME DAMPING}

An alternative statistical acceleration mechanism results from transit-time damping of fluctuations in the magnitude of the magnetic field in the solar wind, as was discussed by Fisk (4). This mechanism was applied by Schwadron et al. (9) to create tails on the distributions of pickup ions, as seen by Ulysses. The physics here is also straightforward. With magnitude fluctuations there is a moving gradient in the magnitude of the magnetic field, which exerts a force and alters the particle speed,

$$
\frac{d v_{\text {parallel }}}{d t} \approx-\frac{v_{\perp}^{2}}{B_{o}} \frac{\partial B}{\partial z}
$$

or, $\left|\Delta v_{\text {parallel }}\right| \approx v_{\perp}^{2} \eta \Delta t / L_{\text {parallel }}$ where $\eta=\delta B / B$, and $L_{\text {parallel }}$ is the characteristic scale length parallel to the mean field.
The gradient, which is produced by a wave, moves parallel to the mean field direction at the parallel phase speed of the wave. Unlike Alfven waves, which have a constant phase speed parallel to the mean field, the magnetosonic waves that generate the magnitude fluctuations have a parallel phase speed that depends on the angle the wave makes with the mean field, or $u_{\text {phase, parallel }}=u_{\text {phase }}\left(L_{\text {parallel }} / L_{\perp}\right)$, where $L_{\perp}$ is the characteristic scale length normal to the mean field. The resulting change in momentum is then the phase speed parallel to the mean field times the change in the particle speed, or

$$
\frac{\Delta p}{p} \approx \frac{u_{\text {phase, parallel }} \Delta v_{\text {parallel }}}{v^{2}}
$$

The resulting diffusion coefficient in momentum is

$$
\frac{D_{p p}}{p^{2}} \approx\left(\frac{v_{\perp}}{v}\right)^{4} \frac{\eta^{2} u_{\text {phase }}^{2}}{v_{\text {parallel }}} \frac{L_{\text {parallel }}}{L_{\perp}^{2}}
$$

It is also possible to do a formal quasi-linear calculation of the diffusion coefficient in momentum, as was done by Fisk (4). The result is qualitatively the same as equation (7). However, the quasi-linear calculation does reveal that the relevant length scale normal to the field, $L_{\perp}$, is not the perpendicular correlation length of the turbulence. Rather, turbulence in the solar wind has a relatively hard power spectrum, generally falling off at less than (wave-number) $^{-2}$. As a result, the level of power falls off less steeply than do the length scales. In a weighted average, then, it is the short length scales that matter most, and, in fact, $L_{\perp}$ is weighted closer to the particle gyro-radius than to the correlation length, which increases the acceleration substantially.

Schwadron et al. (9) performed a study of the tails seen on the distribution functions of interstellar pickup ions, as seen by Ulysses when it was at low latitudes, i.e., in regions where Co-rotating Interaction Regions (CIR) in the solar wind occur. Figure 2, which shows the observed distribution function of interstellar hydrogen, is repeated from Schwadron et al. (9). The quantity $\eta^{2}$ is the square of the ratio of the amplitude of the magnitude fluctuations in the magnetic field to the mean field. The observed distribution function of pickup hydrogen is averaged over 150 days in 1992. The magnitude fluctuations in the field are assumed to be inhomogeneous, with two levels possible, but with the average of the two levels equal to the average observed in the 150-day interval. The diffusion coefficient in momentum space was calculated by Schwadron et al. (9) using the formal quasi-linear 


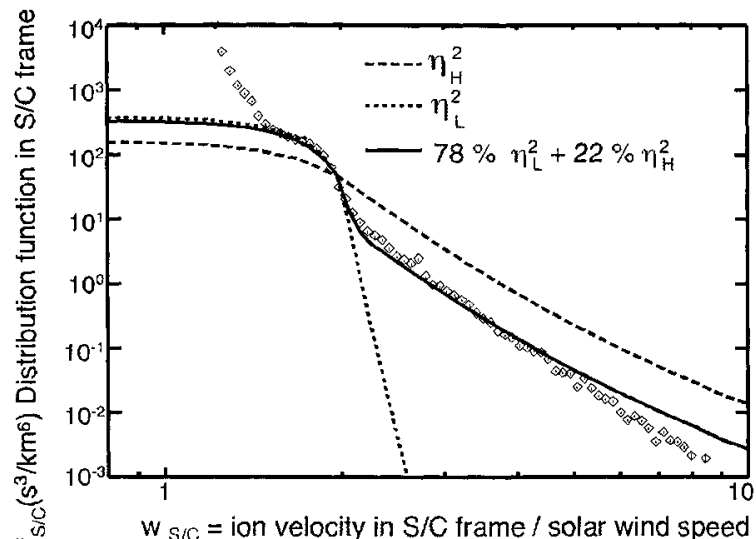

Figure 2. The average proton distribution functions observed by Ulysses/SWICS between day 50 and 200 in 1992 , in the spacecraft reference frame. Model results are plotted in the solid curve (9). The mean value of $\eta^{2}=0.03$.

derivation of Fisk (4). As can be seen in Figure 2, the resulting acceleration yields an excellent fit to the observed tails. The presence of inhomogeneous fluctuations permits a detailed fit to the data, including the knee in the distribution function at twice the solar wind speed. Inhomogeneous turbulence is reasonable over the long time interval used here in particular since turbulence can be expected to be generated within the CIRs itself, but less so in the regions outside the CIR.

Schwadron et al. (9) also did a detailed examination of the correlation of the presence of strong tails on the distribution function with the occurrence of shock waves, e.g., the standing shocks surrounding CIRs. No correlation was found, and indeed the strongest correlation was with enhanced magnitude fluctuations, such as occur interior to the CIR.

Statistical acceleration by transit-time damping is thus a very viable mechanism for producing tails on the distribution function of both solar wind and pickup ions. It is a mechanism which can account in detail for the tails on interstellar pickup ions observed by Ulysses (9) and is a very likely candidate for the tails observed near Earth by ACE, e.g. as seen in Figure 1 (1).

\section{MECHANISMS FOR GENERATING MAGNETIC FIELD MAGNITUDE FLUCTUATIONS}

The strength of statistical acceleration by transittime damping is also its weakness. It is a very efficient acceleration mechanism, and thus presumably will readily damp the magnitude fluctuations that are necessary for a reasonable acceleration rate, i.e. the diffusion coefficient in momentum varies as the square of the amplitude of the field magnitude fluctuations. Indeed, the observed amplitudes of the magnitude fluctuations are quite small, presumably because they are readily damped. As is seen in Figure 2, however, even with small amplitudes, the calculated acceleration rate can account for the observed tails.

To be a viable acceleration process it will be necessary for there to be a continuous source of field magnitude variations. In the case of acceleration in CIRs, as described by Schwadron et al. (9), the streamstream interactions of the solar wind are presumably a continuous source of field magnitude fluctuations. For a continuous source of field magnitude fluctuations near Earth, there are also some possibilities.

There are several arguments that suggest that the footpoints of magnetic field lines random walk in position at the Sun. Jokipii [e.g., (10)] invokes such a random walk to account for cross-field diffusion of energetic particles in the solar wind. Fisk et al. (11) argue that such random walk must occur in the corona that yields slow solar wind. The large scale transport of magnetic flux in the corona in latitude, introduced by Fisk (12), requires a region near the solar equatorial plane where the flux can be transported in longitude, presumably by a reconnection process that in turn yields a random walk of the footpoints. Further, Fisk et al. (13) argue that the emergence of new magnetic flux, in the form of small bipolar loops, and their subsequent reconnection, can account for the acceleration of the solar wind. These loops and their reconnection will also result in random walk of the footpoints.

Inherent in any random walk of field lines is the introduction at the Sun of a component of the field normal to the heliocentric radial direction. As the field line is transported outward with the solar wind, the radial component of the field will decline as $1 / \mathrm{r}^{2}$. The normal component will decline as $1 / r$. Since normal components of various strengths are possible, and thus various angles for field lines are possible, the magnetic strength on adjacent field lines will vary. Equivalently, magnitude variations in the magnetic field in the solar wind are an inherent consequence of the evolution of field lines that random walk back at the Sun. 


\section{CONCLUDING REMARKS}

One of the more interesting recent observations from $\mathrm{ACE}$ has been the ubiquitous presence of pronounced tails on the distribution functions of both solar wind and pickup ions in the slow solar wind. The tails show no correlation with the passage of shock waves, and so appear to be the result of a continuous acceleration in the solar wind. Statistical acceleration is a likely candidate, particularly statistical acceleration by transit-time damping of magnitude fluctuations in the magnetic field. Transit-time damping is a very efficient mechanism; so long as propagating magnitude fluctuations are presence, it should provide any needed acceleration.

Issues remain, however. It will be important to identify the source of the magnitude fluctuations and to show that adequate energy is available to account for the observed tails. It will be important to determine that no other species, e.g. solar wind electrons, are accelerated, such that they would damp and thus remove the available energy in the magnitude fluctuations. It will be important to have a conclusive argument as to why tails occur in the slow solar wind but less so in the fast wind. And finally, as in the case of statistical acceleration in CIRs (9), it will be important to perform detailed fits to observed distribution functions with observed levels of magnetic field magnitude fluctuations.

\section{ACKNOWLEDGEMENTS}

This work was supported, in part, by NASA contract \#NAG5-6912.

\section{REFERENCES}

1. Gloeckler, G., Fisk, L, A., Zurbuchen, T. H., and Schwadron, N. A., in The Acceleration and Transport of Energetic Particles Observed in the Heliosphere, AIP, 2000.

2. Zurbuchen, T. H., Fisk, L. A., Schwadron, N. A., and Gloeckler, G., in The Acceleration and Transport of Energetic Particles Observed in the Heliosphere, AIP, 2000 .

3. Gloeckler, G., Geiss, J., and Fisk, L. A., in Results of the Ulysses Mission, edited by R. Marsden, A. Balogh, and E. Smith, in press, 2000.

4. Fisk, L. A., J. Geophys. Res. 81, 4641 (1976).

5. Barnes, C. W., and Simpson, J. A., Astrophys. J. 210 , L91 (1976).

6. Jokipii, J. R., Phys. Rev. Lett. 26, 666 (1971).

7. Wibberentz, G., and Beuermann, K. P., in Cosmic Plasma Physics, edited by K. Schwindler, New York: Plenum, 1972, pp. 339.

8. Gloeckler, G., Schwadron, N. A., Fisk, L. A., and Geiss, J., Geophys. Res. Lett. 22, 2665 (1995).

9. Schwadron, N. A., Fisk, L. A., and Gloeckler, G., Geophys. Res. Lett. 23, 2871 (1996).

10. Jokipii, J. R., and Parker, E. N., Phys. Rev. Lett. 21, 44 (1969).

11. Fisk,. L. A., Zurbuchen. T. H., and Schwadron, N. A., Astrophys. J. 521, 868 (1999).

12. Fisk, L. A., J. Geophys. Res. 101, 15547 (1996).

13. Fisk, L. A., Schwadron, N. A., and Zurbuchen, T. H., J. Geophys. Res. 104, 19765 (1999). 